\title{
Effets du mode d'irrigation et du contenu en urée de la solution fertilisante sur les caractéristiques de jeunes plants de brocoli, céleri et laitue
}

\author{
$N$ Tremblay ${ }^{1 *}, M$ Senécal ${ }^{2}$ \\ avec la collaboration technique de $M$ Yvon Perron \\ ${ }^{1}$ Agriculture Canada, station de recherches, CP 457, Saint-Jean-sur-Richelieu QC, J3B $6 Z 8$ \\ 2 Gouvernement du Québec, ministère de l'Agriculture des Pêcheries et de l'Alimentation, \\ 867 bd de l'Ange-Gardien, L'Assomption QC, JOK 1GO, Canada
}

(Reçu le 23 janvier 1989; accepté le 11 octobre 1989)

\begin{abstract}
Résumé - On a comparé lirrigation par aspersion à la subirrigation avec des solutions nutritives complètes contenant $0 \%$ ou $50 \%$ de l'azote apporté sous forme d'urée, pour la culture de plants de brocoli (Brassica oleracea $L$ var Italica, cv Emperor), de céleri (Apium graveolens L cv Florida 683) et de laitue (Lactuca sativa L cv Ithaca). L'irrigation par aspersion a favorisé la croissance de la partie aérienne de toutes les espèces mais la présence d'urée fut sans effet significatif. Le mode d'irrigation fut pratiquement sans effet sur la composition minérale du brocoli alors que, chez le céleri et la laitue, les concentrations en azote et en phosphore dans les tissus ont été nettement inférieures avec la subirrigation. Celle-ci a de même généralement appauvri le contenu en éléments de l'extrait du milieu à saturation (SME) des plants de céleri. Selon l'espèce, la présence d'urée a augmenté la concentration en azote, phosphore, potassium, calcium, magnésium, manganèse ou zinc de la partie aérienne. Les résultats témoignent d'importantes différences entre les espèces quant à l'effet du mode d'irrigation et de la présence d'urée.
\end{abstract}

brocoli / céleri / laitue / urée / croissance / composition minérale

Summary - The influence of irrigation method and urea content of fertirrigation solution on vegetable transplants grown in multicellular trays. The influence of overhead vs bottom irrigation in multicellular vegetable transplant production was compared with nutrient solutions containing $0 \%$ or $50 \%$ urea. Broccoli (Brassica oleracea Lvar Italica, cv Emperor), celery (Apium graveolens L cv Florida 683) and lettuce (Lactuca sativa $L$ cv Ithaca) were the species studied. Overhead irrigation promoted shoot growth in all species (table I) but urea did not influence any growth parameters. Irrigation mode was almost of no consequence on mineral composition of broccoli. With celery and lettuce, however, nitrogen and phosphorus concentrations were reduced by bottom irrigation (tables II and III) which for celery, resulted, in low concentrations of nutrients in the saturated medium extract (SME). According to species, urea promoted nitrogen, phosphorus, potassium, calcium, magnesium, manganese or zinc concentrations in shoots. Important differences among species were identified with regard to irrigation or urea treatments.

broccoli / celery / lettuce / urea / growth / mineral composition

\section{INTRODUCTION}

L'élevage des plants maraîchers en minimottes est une technique en expansion. II pose des problèmes d'alimentation minérale et hydrique. L'irrigation par aspersion a d'abord été proposée en raison de sa répartition uniforme. Mais, pour limiter la prolifération des maladies, l'apport souter- rain a été également préconisé. La fertilisation foliaire a été appliquée depuis longtemps sur les espèces fruitières arbustives sans convaincre l'ensemble des techniciens. De nombreux essais se sont révélés assez positifs sur de jeunes plants d'espèces herbacées, même (Nowak, 1986) lorsque la fertilisation conventionnelle est optimale. Cependant, des phénomènes de toxici-

\footnotetext{
* Correspondance et tirés à part.
} 
té (brûlure du feuillage, diminution de la capacité photosynthétique) consécutifs à des applications de solutions nutritives complètes ont été rapportés (Kannan, 1986).

Par ailleurs, l'utilisation de l'urée dans les solutions nutritives est sujette à controverse. L'absorption de l'urée par les feuilles est plus rapide que celle du N-NH${ }_{4}$ ou du N-NO 3 (Marschner, 1986) ou de n'importe quel autre anion inorganique (Swietlik et Faust, 1984). L'urée est absorbée, métabolisée et transportée très rapidement après l'application (Ferree et Cahoon, 1987). Sa présence semble favoriser la pénétration d'autres ions en induisant des changements chimiques dans les membranes (Kannan, 1986). Toutefois, l'urée peut subir une dégradation rapide en $\mathrm{N}$ $\mathrm{NH}_{4}$ et entraîner des réactions de toxicité (Alexander et Schroeder, 1987; Zehnalek et Minar, 1987). Aussi, son utilisation a été déconseillée pour les plantes en pots; les effets négatifs étant cependant plus accentués en laine de roche qu'en substrat à base de tourbe (Klougart, 1976). Enfin l'application simultanée des formes urée, $\mathrm{N}-\mathrm{NH}_{4}$ et $\mathrm{N}-\mathrm{NO}_{3}$ facilite l'absorption des cations fer et zinc par les feuilles (Alexander et Schroeder, 1987; Reed, 1988).

Le premier objectif de cette recherche est de comparer, quant à leurs effets sur la croissance et la nutrition, 2 façons d'appliquer la solution nutritive en usage dans les cultures commerciales de plants maraîchers en minimottes. Le second objectif est d'évaluer, selon les mêmes critères, l'influence de la présence d'urée dans la solution nutritive et de vérifier si son effet est le même selon les modes d'irrigation de la solution.

\section{MATÉRIEL ET MÉTHODES}

\section{Conditions expérimentales}

Les espèces suivantes : brocoli (Brassica oleracea $L$ var Italica, cv. Emperor), céleri (Apium graveolens L cv. Florida 683) et laitue (Lactuca sativa L cv Ithaca) sont semées dans des plateaux alvéolés de polystyrène (Speedling Inc.) contenant un substrat à base de mousse de tourbe (Metro Mix 200, WR Grace \& Co du Canada Ltée, Ajax, Ontario). L'analyse de l'extrait du milieu à saturation [Saturated medium extract $=\mathrm{SME}$ (Warncke, 1986)], tel qu'il se présente avant le semis, révèle les valeurs suivantes de conductivité électrique et de concentrations en éléments nutritifs : $2,3 \mathrm{dS} \cdot \mathrm{m}^{-1}$, et (en mg.t-1 $) ; 8 \mathrm{~N}-\mathrm{NH}_{4}, 159 \mathrm{~N}-\mathrm{NO}_{3}, 36 \mathrm{P}, 382 \mathrm{~K}, 535$ $\mathrm{Ca}, 463 \mathrm{Mg}, 4 \mathrm{Fe}, 4 \mathrm{Mn}, 0,4 \mathrm{Cu}$ et $2 \mathrm{Zn}$, avec un $\mathrm{pH}$ de 6,35 , mesuré directement dans la pâte de substrat saturé. Chaque unité expérimentale pour le brocoli et la laitue est constituée de 20 alvéoles contenant chacune un plant, et provenant de plateaux de modèle $125\left(15,5 \mathrm{~cm}^{3} /\right.$ alvéole). Pour le céleri, l'unité expérimentale comprend 36 alvéoles du plateau modèle
080A ( $8,5 \mathrm{~cm}^{3} /$ alvéole). Les semis sont faits le 7 janvier pour le céleri et le 13 janvier 1988 pour le brocoli et la laitue. Par la suite, les plants sont disposés dans une enceinte climatisée de $3,3 \mathrm{~m}^{2}$. La température de l'air est fixée à $22^{\circ} \mathrm{C}$ le jour et $16^{\circ} \mathrm{C}$ la nuit. L'humidité relative est de $85 \%$. La photopériode est de $18 \mathrm{~h}$ (5h00 - 23h00). L'éclairage fournit une densité de flux photonique de $295 \pm s=18 \mu \mathrm{mol} \cdot \mathrm{m}^{-2} \cdot \mathrm{s}^{-1}$, avec des tubes fluorescents Cool-White et des ampoules incandescentes dans un rapport de puissance à l'entrée de $5 / 1$. Le substrat est gardé uniformément humide par un arrosage à l'eau distillée jusqu'à l'émergence des plantules. Les traitements débutent à la levée et sont appliqués tous les jours vers 16 h00 sauf le dimanche. Les plants de tous les traitements reçoivent un arrosage par aspersion avec de l'eau distillée vers 8 h00 le matin.

\section{Traitements}

Les traitements d'irrigation se font selon 2 méthodes : - par aspersion à l'aide d'un arrosoir (irrigation par aspersion), ou

- par trempage de la partie radiculaire seulement (subirrigation). Dans les 2 cas, on utilise une quantité suffisante de solution pour saturer complètement le substrat. Les traitements de fertilisation sont les suivants :

- aucun apport d'urée : la nutrition azotée est fournie sous 3 formes dans les proportions: $\left[\mathrm{N}-\mathrm{NO}_{3}(2) \mathrm{N}-\mathrm{NH}_{4}(1), \mathrm{N}\right.$-Urée (0)] ou :

- apport d'urée à $50 \%$ de la dose totale d'azote $\left[\mathrm{N}-\mathrm{NO}_{3}(2), \mathrm{N}-\mathrm{NH}_{4}(1), \mathrm{N}\right.$-Urée (3)].

Les 4 traitements sont constitués des combinaisons factorielles des 2 traitements d'irrigation et des 2 pourcentages d'urée $\left(2^{2}\right)$. Les 2 solutions apportent les mêmes concentrations en éléments nutritifs $\left(\mathrm{mg} \cdot \mathrm{l}^{-1}\right)$ soit : $350 \mathrm{~N}, 40 \mathrm{P}, 200 \mathrm{~K}, 50 \mathrm{Ca}, 20 \mathrm{Mg}, 2 \mathrm{Fe}$ (sous la forme du chélate EDTA), $0,5 \mathrm{Mn}, 0,03 \mathrm{Cu}, 0,02 \mathrm{Mo}$ et $0,05 \mathrm{Zn}$. Le pH des solutions est ajusté à 5,5 par du $\mathrm{NaOH}$. Tous les plateaux sont retirés de l'enceinte climatisée pour recevoir les traitements et y sont replacés aléatoirement par la suite.

\section{Echantillonnage et mesures}

La partie foliaire est rincée par une application d'eau distillée avant l'échantillonnage. Les paramètres de croissance sont mesurés $21 \mathrm{j}$ après le semis pour la laitue, $23 \mathrm{j}$ pour le brocoli et $40 \mathrm{j}$ pour le céleri. Des échantillons de 6 plants de brocoli et de laitue, de 16 plants de céleri sont prélevés hors des bordures du plateau. Les feuilles sont comptées et leur surface mesurée avec un intégrateur de surface de modèle LiCor Li-3100 (Li-Cor, Lincoln, $\mathrm{Ne}$ ). La partie aérienne est sectionnée au niveau du substrat pour toutes les espèces. La partie radiculaire du brocoli et de la laitue est dissociée du substrat dans un bain d'eau courante. Afin de permettre la comparaison avec des travaux antérieurs (Tremblay et Gosselin 1989a, b), et pour le céleri seulement, on introduit 16 alvéoles contenant le substrat et les racines dans un entonnoir Buchner et l'on sature d'eau distillée durant $2 \mathrm{~h}$.

La mesure du $\mathrm{pH}$ est faite directement dans la pâte de substrat saturé et, par la suite, une succion est appliquée pour recueillir l'extrait du milieu à saturation 
(SME). La conductivité électrique est mesurée sur cet extrait. Les racines sont ensuite récupérées du substrat dans un bain d'eau courante. Les parties aériennes et radiculaires sont séchées au four à $70^{\circ} \mathrm{C}$ et pesées. Elles sont ensuite broyées et soumises à une digestion aux fins d'analyses minérales selon la méthode de Isaac et Johnson (1976). La teneur en N et en $\mathrm{P}$ des tissus est déterminée par la méthode Technicon Auto-Analyser II-C (No. 334-74 W/B+) et les concentrations en $\mathrm{K}, \mathrm{Ca}, \mathrm{Mg}, \mathrm{B}, \mathrm{Cu}, \mathrm{Fe}, \mathrm{Mn}$ et $\mathrm{Zn}$ par spectrométrie d'émission atomique au plasma (ICP. AES). Les teneurs en $\mathrm{N}-\mathrm{NO}_{3}, \mathrm{~N}-\mathrm{NH}_{4}$ et en urée du SME sont mesurées par la méthode Technicon AutoAnalyser No. 487-77A, No. 334-74 W/B+ et No. 600$80 \mathrm{~A}$, respectivement.

L'analyse de variance est effectuée selon un dispositif complètement aléatoire à 5 répétitions. Afin d'affranchir les résultats de composition minérale de l'effet des traitements sur le pourcentage de matière sèche (Bates, 1971; Beaufils, 1973), nous avons effectué une analyse de covariance sur les analyses tissulaires avec le pourcentage de matière sèche de l'échantillon comme covariée, laquelle s'est effectivement révélée très significative dans la plupart des cas. Les moyennes rapportées aux tableaux II, III et IV sont donc celles ajustées pour la covariée et représentent l'effet des traitements sur la composition minérale des tissus ramenée à une valeur uniforme du pourcentage de matière sèche.

\section{RÉSULTATS ET DISCUSSION}

\section{Effets du mode d'irrigation}

Nos résultats montrent une accumulation supérieure de masse sèche mesurée sur la partie aérienne de toutes les espèces irriguées par aspersion (tableau I), ce qui confirme, dans le cas de la laitue, les travaux de Kratky et Mishima
(1981). Le mode d'irrigation n'a pas d'effet sur la matière sèche des racines. L'aspersion réduit le pourcentage de matière sèche du système aérien. La subirrigation favorise le rapport de matière sèche partie radiculaire : partie aérienne chez le céleri et la laitue seulement.

L'influence du mode d'irrigation de la solution nutritive varie avec l'espèce. En effet, le céleri montre une croissance de la partie aérienne nettement supérieure sous aspersion (tableau I). Le brocoli semble toutefois avoir moins réagi lorsqu'on le compare à la laitue. La présence de cires cuticulaires et épicuticulaires peut entraver l'absorption foliaire (Swietlik et Faust, 1984).

La subirrigation, chez le brocoli, n'a eu d'effet que sur les racines dont le contenu en zinc a augmenté (tableaux II et III). Chez le céleri, les teneurs en azote et phosphore ont été inférieures dans les parties aérienne et radiculaire. Les teneurs en potassium et magnésium dans les racines et en calcium, magnésium (tableau II) et zinc (tableau III) dans les parties aériennes ont été supérieures. On a mesuré également une teneur réduite en bore dans les racines. Dans le cas de la laitue, la subirrigation a réduit les concentrations en azote dans toutes les parties et en phosphore dans la partie aérienne seulement (tableau II). Les éléments potassium, calcium, magnésium, cuivre et fer n'ont pas été affectés par les traitements d'irrigation. La concentration en bore dans les parties aériennes a toutefois été diminuée alors que le manganèse a été haussé de façon importante (tableau III). La subirrigation a accru le contenu en zinc des racines de laitue.

Tableau I. Influence du mode d'irrigation en culture de semis sur les paramètres végétatifs de brocoli, céleri et laitue cultivés en minimottes. Effets significatifs aux seuils $5 \%\left({ }^{\star}\right), 1 \%\left({ }^{* \star}\right)$ ou $0,1 \%\left(^{\star \star \star}\right)$ de l'analyse de variance.

\begin{tabular}{|c|c|c|c|c|c|c|}
\hline Espèce & Application & $\begin{array}{l}\text { Surface foliaire } \\
\left.\text { ( } \mathrm{cm}^{2} / \text { plant }\right)\end{array}$ & $\begin{array}{l}\text { m.s. P. aér. } \\
\text { (mg/plant) }\end{array}$ & $\begin{array}{c}\% \text { m.s. } \\
\text { P. aérięne }\end{array}$ & $\begin{array}{c}\text { m.s. racines } \\
\text { (mg/plant) }\end{array}$ & $\begin{array}{l}\text { Ratio m.s. } \\
\text { P. rac : P. aér. }\end{array}$ \\
\hline \multirow{2}{*}{ Brocoli } & Aspersion & 78 & 406 & 9.4 & 134 & 0,33 \\
\hline & Subirrigation & 62 & 353 & 10,5 & $\begin{array}{l}132 \\
\text { NS }\end{array}$ & $\begin{array}{c}0,37 \\
\text { NS }\end{array}$ \\
\hline \multirow{2}{*}{ Céleri } & Aspersion & 37 & 152 & 8.5 & 37 & 0,24 \\
\hline & Subirrigation & 21 & 93 & $\underset{* * *}{11,3}$ & $\begin{array}{l}35 \\
\text { NS }\end{array}$ & $\underset{x+*}{0,37}$ \\
\hline \multirow{2}{*}{ Laitue } & Aspersion & 118 & 247 & 4,5 & 35 & 0,14 \\
\hline & Subirrigation & 90 & 207 & 5,1 & $\begin{array}{l}38 \\
\text { NS }\end{array}$ & 0,18 \\
\hline
\end{tabular}


Tableau II. Influence du mode d'irrigation en culture de semis sur le contenu en éléments majeurs [g.100 $\left.\mathrm{g}^{-1}(\mathrm{MS})\right]$ des parties aériennes et radiculaires de brocoli, céleri et laitue. Effets significatifs aux seuils $5 \%\left({ }^{\star}\right), 1 \%\left(^{\star \star}\right)$ ou $0,1 \%\left(^{\star \star \star}\right)$. Analyse de covariance.

\begin{tabular}{|c|c|c|c|c|c|c|c|c|c|c|c|}
\hline \multirow[t]{2}{*}{ Espèce } & \multirow[t]{2}{*}{ Irrigation } & \multicolumn{2}{|c|}{$N$} & \multicolumn{2}{|c|}{$\mathrm{P}$} & \multicolumn{2}{|c|}{$\mathrm{K}$} & \multicolumn{2}{|c|}{$\mathrm{Ca}$} & \multicolumn{2}{|c|}{$\mathrm{Mg}$} \\
\hline & & Aér. & Rac. & Aér. & Rac. & Aér. & Rac. & Aér. & Rac. & Aér. & Rac. \\
\hline & & 一 & - & 一 & - & - & 一 & - & - & - & - \\
\hline \multirow[t]{2}{*}{ Brocoli } & $\begin{array}{l}\text { Aspersion } \\
\text { Subirrigation }\end{array}$ & $\begin{array}{l}4,18 \\
3,95\end{array}$ & $\begin{array}{l}2,21 \\
1,97\end{array}$ & $\begin{array}{l}0,51 \\
0,50\end{array}$ & $\begin{array}{l}0,33 \\
0,28\end{array}$ & $\begin{array}{l}3,75 \\
3,67\end{array}$ & $\begin{array}{l}2,19 \\
2,25\end{array}$ & $\begin{array}{l}1,30 \\
1,26\end{array}$ & $\begin{array}{l}0,71 \\
0,72\end{array}$ & $\begin{array}{l}0,47 \\
0,45\end{array}$ & $\begin{array}{l}2,12 \\
2,07\end{array}$ \\
\hline & & NS & NS & NS & NS & NS & NS & NS & NS & NS & NS \\
\hline \multirow[t]{2}{*}{ Céleri } & $\begin{array}{l}\text { Aspersion } \\
\text { Subirrigation }\end{array}$ & $\begin{array}{l}3,92 \\
2,68\end{array}$ & $\begin{array}{l}4,06 \\
2,29\end{array}$ & $\begin{array}{l}0,46 \\
0,34\end{array}$ & $\begin{array}{l}0,53 \\
0,40\end{array}$ & $\begin{array}{l}3,49 \\
3,41\end{array}$ & $\begin{array}{l}2,09 \\
3,39\end{array}$ & $\begin{array}{l}0,71 \\
1,41\end{array}$ & $\begin{array}{l}1,11 \\
0,69\end{array}$ & $\begin{array}{l}0,33 \\
0,45\end{array}$ & $\begin{array}{l}0,45 \\
0,60\end{array}$ \\
\hline & & **** & $\ldots$ & * & $* * *$ & NS & $\cdots$ & $\cdots$ & NS & $\cdots$ & "* \\
\hline \multirow[t]{2}{*}{ Laitue } & $\begin{array}{l}\text { Aspersion } \\
\text { Subirrigation }\end{array}$ & $\begin{array}{l}4,45 \\
3,42\end{array}$ & $\begin{array}{l}2,43 \\
1,87\end{array}$ & $\begin{array}{l}0,59 \\
0,48\end{array}$ & $\begin{array}{l}0,49 \\
0,35\end{array}$ & $\begin{array}{l}4,52 \\
4,58\end{array}$ & $\begin{array}{l}1,82 \\
2,20\end{array}$ & $\begin{array}{l}0,68 \\
0,65\end{array}$ & $\begin{array}{l}5,93 \\
7,50\end{array}$ & $\begin{array}{l}0,45 \\
0,44\end{array}$ & $\begin{array}{l}1,26 \\
1,31\end{array}$ \\
\hline & & *.** & * & $* * *$ & NS & NS & NS & NS & NS & NS & NS \\
\hline
\end{tabular}

Tableau III. Influence du mode d'irrigation en culture de semis sur le contenu en éléments mineurs [ $\mu \mathrm{g} \cdot \mathrm{g}^{-1}$ (MS)] des parties aériennes et radiculaires de brocoli, céleri et laitue. Effets significatifs aux seuils $5 \%\left({ }^{*}\right), 1 \%\left(\left(^{* *}\right)\right.$ ou $0,1 \%\left(^{\star * *}\right)$. Analyse de covariance.

\begin{tabular}{|c|c|c|c|c|c|c|c|c|c|c|c|}
\hline \multirow[t]{2}{*}{ Espèce } & \multirow[t]{2}{*}{ Irrigation } & \multicolumn{2}{|c|}{ B } & \multicolumn{2}{|c|}{$\mathrm{Cu}$} & \multicolumn{2}{|c|}{$\mathrm{Fe}$} & \multicolumn{2}{|c|}{$M n$} & \multicolumn{2}{|c|}{$\mathrm{Zn}$} \\
\hline & & Aér. & Rac. & Aér. & Rac. & Aér. & Rac. & Aér. & Rac. & Aér. & Rac. \\
\hline & & - & - & - & - & - & - & - & - & - & - \\
\hline Brocoli & $\begin{array}{l}\text { Aspersion } \\
\text { Subirrigation }\end{array}$ & $\begin{array}{l}69 \\
53 \\
\text { NS }\end{array}$ & $\begin{array}{l}28 \\
31 \\
\text { NS }\end{array}$ & $\begin{array}{c}5 \\
9 \\
\text { NS }\end{array}$ & $\begin{array}{l}39 \\
38 \\
\text { NS }\end{array}$ & $\begin{array}{r}108 \\
87 \\
\text { NS }\end{array}$ & $\begin{array}{c}992 \\
857 \\
\text { NS }\end{array}$ & $\begin{array}{l}40 \\
25 \\
\text { NS }\end{array}$ & $\begin{array}{r}149 \\
144 \\
\text { NS }\end{array}$ & $\begin{array}{l}22 \\
23 \\
\text { NS }\end{array}$ & $\begin{array}{l}37 \\
44 \\
\cdots\end{array}$ \\
\hline Céleri & $\begin{array}{l}\text { Aspersion } \\
\text { Subirrigation }\end{array}$ & $\begin{array}{l}38 \\
46 \\
\text { NS }\end{array}$ & $\begin{array}{l}44 \\
37 \\
. \cdot\end{array}$ & $\begin{array}{c}7 \\
9 \\
\text { NS }\end{array}$ & $\begin{array}{l}20 \\
31 \\
\text { NS }\end{array}$ & $\begin{array}{l}93 \\
79 \\
\text { NS }\end{array}$ & $\begin{array}{c}157 \\
121 \\
\text { NS }\end{array}$ & $\begin{array}{l}46 \\
47 \\
\text { NS }\end{array}$ & $\begin{array}{r}221 \\
194 \\
\text { NS }\end{array}$ & $\begin{array}{l}12 \\
17 \\
*\end{array}$ & $\begin{array}{l}29 \\
29 \\
\text { NS }\end{array}$ \\
\hline Laitue & $\begin{array}{l}\text { Aspersion } \\
\text { Subirrigation }\end{array}$ & $\begin{array}{l}37 \\
31 \\
*\end{array}$ & $\begin{array}{l}22 \\
26 \\
\text { NS }\end{array}$ & $\begin{array}{c}8 \\
10 \\
\text { NS }\end{array}$ & $\begin{array}{l}39 \\
37 \\
\text { NS }\end{array}$ & $\begin{array}{r}287 \\
409 \\
\text { NS }\end{array}$ & $\begin{array}{c}539 \\
570 \\
\text { NS }\end{array}$ & $\begin{array}{l}37 \\
83 \\
\ldots *\end{array}$ & $\begin{array}{r}113 \\
150 \\
\text { NS }\end{array}$ & $\begin{array}{l}39 \\
48 \\
\text { NS }\end{array}$ & $\begin{array}{l}33 \\
56 \\
\ldots\end{array}$ \\
\hline
\end{tabular}

\section{Effets de l'urée}

La proportion d'urée dans la solution fertilisante n'a eu aucun effet significatif sur les paramètres végétatifs mesurés; ni seule, ni en interaction avec le mode d'application de la solution. Conover et Poole (1986) ainsi que Kannan (1986) ont conclu que la source d'azote n'a pas beaucoup d'importance dans le cas de plusieurs espèces.

L'utilisation de solutions nutritives contenant de l'urée n'a donné lieu à aucune réaction de toxicité. Nous pouvons supposer sur la base des travaux d'Elliott (1986) que l'urée fut rapidement hydrolysée en $\mathrm{N}-\mathrm{NH}_{4}$ dans le milieu de croissance. Or, le $\mathrm{N}-\mathrm{NH}_{4}$ peut provoquer des réactions de toxicité (Alexander et Schroeder, 1987). Selon Magalhaes et Wilcox (1984), toutefois, les milieux tourbeux conviennent très bien à l'utilisation de $\mathrm{N}-\mathrm{NH}_{4}$ en procurant une croissance égale et même supérieure à celle obtenue avec la forme $\mathrm{N}-\mathrm{NO}_{3}$, ce que tendent à confirmer nos résultats.

La présence d'urée n'a affecté la composition minérale des racines d'aucune espèce; ce qui confirme, pour l'azote, les résultats rapportés par Swietlik et Faust (1984). Chez aucune espèce les concentrations en bore et en fer dans la partie aérienne n'ont été affectées par la présence d'urée. La présence d'urée a toutefois donné lieu à des concentrations accrues de certains éléments dans les parties aériennes (tableau IV). C'est le cas avec l'azote, le phosphore, le potassium et le zinc chez le brocoli; le calcium, le magnésium et le manganèse chez le céleri ainsi 
Tableau IV. Influence du contenu en urée de la solution nutritive en culture de semis sur le contenu en éléments des parties aériennes de brocoli, céleri et laitue. Effets significatifs aux seuils $5 \%\left(^{*}\right), 1 \%\left(^{* *}\right)$ ou $0,1 \%\left(^{* * *}\right)$. Analyse de covariance.

\begin{tabular}{|c|c|c|c|c|c|c|c|c|}
\hline Espèce & Urée & $\underset{\left(g \cdot 100 g^{-1}\right)}{N}$ & $\begin{array}{c}P \\
\left(g^{\cdot} 100 g^{-1}\right)\end{array}$ & $\begin{array}{c}K \\
\left(g^{\prime} \cdot 100 \mathrm{~g}^{-1}\right)\end{array}$ & $\begin{array}{c}\mathrm{Ca} \\
\left(\mathrm{g}^{\cdot}+00 \mathrm{~g}^{-1}\right)\end{array}$ & $\underset{\left(\mathrm{g} \cdot 100 \mathrm{~g}^{-1}\right)}{\mathrm{Mg}}$ & $\underset{\left(\mu \mathrm{g} \cdot \mathrm{g}^{-1}\right)}{\mathrm{Mn}}$ & $\underset{\left(\mu \mathrm{Zn} \cdot \mathrm{g}^{-1}\right)}{\mathrm{Zn}}$ \\
\hline \multirow[t]{2}{*}{ Brocoli } & $\begin{array}{r}0 \% \\
50 \%\end{array}$ & $\begin{array}{l}3,95 \\
4,18\end{array}$ & $\begin{array}{l}0,48 \\
0,53\end{array}$ & $\begin{array}{l}3,36 \\
4,06\end{array}$ & $\begin{array}{l}1,31 \\
1,26\end{array}$ & $\begin{array}{l}0,47 \\
0,44\end{array}$ & $\begin{array}{l}34 \\
31\end{array}$ & $\begin{array}{l}21 \\
25\end{array}$ \\
\hline & & $* *$ & $\cdots$ & $\cdot$ & NS & NS & NS & * \\
\hline \multirow[t]{2}{*}{ Céleri } & $\begin{array}{r}0 \% \\
50 \%\end{array}$ & $\begin{array}{l}3,27 \\
3,33\end{array}$ & $\begin{array}{l}0,39 \\
0,41\end{array}$ & $\begin{array}{l}3,41 \\
3,50\end{array}$ & $\begin{array}{l}1,01 \\
1,11\end{array}$ & $\begin{array}{l}0,37 \\
0,40\end{array}$ & $\begin{array}{l}45 \\
48\end{array}$ & $\begin{array}{l}14 \\
14\end{array}$ \\
\hline & & NS & NS & NS & $*$ & $\cdots$ & * & NS \\
\hline \multirow[t]{2}{*}{ Laitue } & $\begin{array}{r}0 \% \\
50 \%\end{array}$ & $\begin{array}{l}3,83 \\
4,05\end{array}$ & $\begin{array}{l}0,52 \\
0,55\end{array}$ & $\begin{array}{l}4,54 \\
4,55\end{array}$ & $\begin{array}{l}0,63 \\
0,68\end{array}$ & $\begin{array}{l}0,45 \\
0,44\end{array}$ & $\begin{array}{l}51 \\
68\end{array}$ & $\begin{array}{l}40 \\
47\end{array}$ \\
\hline & & $\star *$ & $*$ & NS & * & NS & $*$ & * \\
\hline
\end{tabular}

qu'avec l'azote, le phosphore, le calcium, le manganèse et le zinc chez la laitue. Kannan (1986) rapporte que la source d'azote n'a pas d'effet sur la composition en azote de feuilles de pommiers. Nos résultats confirment cette observation dans le cas du céleri mais l'infirment pour le brocoli et la laitue. Ferree et Cahoon (1987) ont noté un effet positif des applications foliaires d'urée sur les concentrations en calcium et fer de feuilles de pommiers. Swietlik et Faust (1984) ont fait mention de travaux où l'apport d'urée a avantagé l'absorption du manganèse, tout en n'ayant aucun effet notable sur l'absorption du phosphore et du magnésium.

Généralement, cet effet de l'urée s'est exercé au même titre sur les plants soumis à la subirrigation des fertilisants que sur ceux soumis à l'irrigation par aspersion. Cette observation tend à confirmer l'opinion que les mécanismes d'absorption des ions par voie foliaire sont semblables à ceux qui gouvernent l'absorption par les racines
(Kannan, 1986). Toutefois, le contenu en manganèse des parties foliaires du céleri s'est accru en présence d'urée, surtout avec la subirrigation (Interaction irrigation $\mathrm{x} \%$ urée significative à $P \leq 0,032)$. La concentration de la partie aérienne en cuivre chez le céleri et la laitue a décru en présence d'urée lorsqu'une irrigation par aspersion était faite, mais s'est vue favorisée par la subirrigation [Interaction irrigation $x \%$ urée significative à $P \leq 0,036$ (céleri) et $P \leq 0,021$ (laitue)]. Il en fut ainsi pour le zinc chez la partie aérienne de la laitue (Interaction irrigation $\times \%$ urée significative à $P \leq 0,004$ ).

\section{Céleri : Analyses SME}

La subirrigation a élevé le $\mathrm{pH}$ de la pâte saturée (tableau V). En revanche, la conductivité électrique et les valeurs de concentration en éléments nutritifs du SME ont été beaucoup plus

Tableau V. Influence du mode d'irrigation et du contenu en urée de la solution nutritive en culture de semis de céleri sur le $\mathrm{pH}$, la conductivité ( $\mathrm{dS} \cdot \mathrm{m}^{-1}$ ) et le contenu en éléments (mg.litre $\left.{ }^{-1}\right)$ de l'extrait saturé du milieu nutritif. Effets significatifs aux seuils $5 \%\left(^{\star}\right), 1 \%\left(^{\star \star}\right)$ ou $0,1 \%\left(^{\star \star \star}\right)$ de l'analyse de variance.

\begin{tabular}{|c|c|c|c|c|c|c|c|c|c|c|}
\hline Traitement & $\mathrm{pH}$ & Cond. & $\mathrm{N}-\mathrm{NH}_{4}$ & $\mathrm{~N}-\mathrm{NO}_{3}$ & $\mathrm{P}$ & $\mathrm{K}$ & $\mathrm{Ca}$ & $\mathrm{Mg}$ & $\mathrm{B}$ & $\mathrm{Fe}$ \\
\hline Aspersion & 5,05 & 0,30 & 8 & 14 & 4 & 37 & 22 & 13 & 1,1 & 1,2 \\
\hline \multirow[t]{2}{*}{ Subirrigation } & 5,86 & 0,12 & 2 & 1 & 1 & 23 & 13 & 8 & 0,6 & 1,2 \\
\hline & $\cdots$ & $* * *$ & $* * *$ & $* *$ & $* * *$ & $* * *$ & *** & $\ldots$ & $\ldots$ & NS \\
\hline $0 \%$ urée & 5,20 & 0,22 & 5 & 9 & 3 & 31 & 15 & 10 & 0,9 & 1,3 \\
\hline \multirow[t]{2}{*}{$50 \%$ urée } & 5,40 & 0,19 & 6 & 6 & 2 & 28 & 19 & 12 & 0,9 & 1,0 \\
\hline & $*$ & NS & NS & NS & NS & NS & * & * & NS & . \\
\hline
\end{tabular}


faibles avec ce traitement, sauf pour le fer qui n'a pas été affecté. II est possible que l'élévation du $\mathrm{pH}$ à la suite de subirrigation soit due à une absorption plus complète du $\mathrm{N}-\mathrm{NO}_{3}$ que dans le cas de l'irrigation par aspersion. En effet, avec cette dernière, la quantité de $\mathrm{N}-\mathrm{NO}_{3}$ restée dans le SME est plus grande que celle de $\mathrm{N}-\mathrm{NH}_{4}$, ce qui témoigne, soit d'une plus grande nitrification, soit - ce qui est plus probable - d'une absorption préférentielle du $\mathrm{N}-\mathrm{NH}_{4}$ par le céleri (Tremblay et Gosselin, 1989a). Si l'on considère les concentrations optimales rapportées par Kirven (1986) et le fait que la quantité initiale d'azote dans les solutions nutritives était de $350 \mathrm{mg} \cdot \mathrm{l}^{-1}$, on peut supposer que les plants soumis à la subirrigation ont vraisemblablement été en situation de carence d'azote. Les plants sous le traitement d'aspersion, bien que plus développés, ont en effet moins épuisé les réserves du milieu nutritif, ce qui suggère qu'une partie significative du $\mathrm{N}$ requis pour leur croissance a été dérivée des processus de l'absorption foliaire. Ils ont pu sélectionner en priorité le $\mathrm{N}-\mathrm{NH}_{4}$ du milieu nutritif, laissant la quantité de $\mathrm{N}-\mathrm{NO}_{3}$ pour compte.

La présence d'urée à raison de $50 \%$ de la dose d'azote a donné un $\mathrm{pH}$ de la pâte saturée et une concentration en calcium et magnésium du SME supérieurs. Un effet similaire de l'urée sur le $\mathrm{pH}$ de substrats servant à la production de plantes vertes a été noté (Conover et Poole, 1986). Cette hausse du pH provient de la libération d'ions $\mathrm{OH}^{-}$consécutive à la libération d'ammoniac par l'urée et à son association à l'eau du substrat pour former l'ion $\mathrm{N}^{-\mathrm{NH}_{4}^{+}}$(Klougart, 1976), et témoigne d'une hydrolyse de l'urée. A l'inverse, la concentration en fer s'est trouvée légèrement plus faible. La concentration en cuivre n'a pas été affectée par les traitements et celles en manganèse et zinc ont démontré des variances non homogènes rendant inapplicables les tests de l'analyse de variance.

\section{CONCLUSION}

D'après nos résultats, il est manifeste que des différences importantes existent entre les espèces quant aux effets des traitements sur la croissance et sur l'absorption minérale. Ces différences peuvent s'expliquer par des raisons anatomiques, comme la quantité et la distribution des stomates sur les faces de la feuille (Kannan, 1986), la composition et les caractéristiques anatomiques de la cuticule qui, plus que son épaisseur, interviennent dans la pénétration des éléments nutritifs (Swietlik et Faust, 1984). II apparaît que, à un degré qui varie selon l'espèce, l'apport des solutions fertilisantes par la méthode conventionnelle de l'aspersion favorise la croissance de la partie aérienne. Toutefois, la subirrigation se révèle un moyen de contrôler la croissance végétative, et également d'augmenter le pourcentage de matière sèche des plants et donc de les endurcir en prévision de leur transport sur de grandes distances ou de leur plantation dans des conditions climatiques difficiles. Comme en témoignent nos résultats sur le céleri, ce ralentissement de croissance observé chez les 3 espèces semble lié à un approvisionnement réduit en azote et phosphore. Ces éléments, lorsqu'ils ne sont pas apportés par voie foliaire, semblent rapidement épuisés dans le substrat. Cette hypothèse est confirmée par le fait que le rapport de matière sèche racines : partie aérienne s'est accru avec la subirrigation en l'absence d'effets significatifs sur la matière sèche des racines; ce qui témoigne d'un phénomène de carence minérale (Wilson, 1988). En effet, les concentrations en azote et phosphore chez le céleri, et dans une moindre mesure chez la laitue, ont été diminuées par la subirrigation.

L'utilisation d'urée a favorisé l'absorption des éléments nutritifs dans la partie aérienne sans égard au mode d'irrigation, sauf pour les interactions déjà mentionnées. Cette influence bénéfique ne s'est toutefois exprimée de façon significative que pour certains éléments et selon l'espèce. Dans le cadre de cette expérience, l'urée s'avère donc une source d'azote intéressante pour la production de plants maraîchers.

\section{RÉFÉRENCES}

Alexander A, Schroeder M (1987) Modern trends in foliar fertilization. J Plant Nutr 10, $1391-1399$

Bates TE (1971) Factors affecting critical nutrient concentrations in plants and their evaluation: $A$ review. Soil Sci 112, 116-130

Beaufils ER (1973) Diagnosis and recommendation integrated system (DRIS). Soil Sci Bull 1, Department of soil science and agrometeorology, University of Natal Pietermaritzburg, South Africa, $132 p$

Conover CA, Poole RT (1986) Nitrogen source effects on growth and tissue content of selected foliage plants. Hort Science 21, $1008-1009$

Elliott GC (1986) Urea hydrolysis in potting media. $J$ Am Soc Hortic Sci 111, 862-866

Ferree DC, Cahoon GA (1987) Influence of leaf to fruit ratios and nutrient sprays on fruiting, mineral elements, and carbohydrates of apple trees. $J A m$ Soc Hortic Sci 112, 445-449

Isaac RA, Johnson WC (1976) Determination of total nitrogen in plant tissue using a block digestor. $J$ Assoc Off Anal Chem 59, 98-100

Kannan S (1986) Foliar absorption and transport of inorganic nutrients. CRC Crit Rev Plant Sci 4, 341-375 
Kirven DM (1986) An industry viewpoint: Horticultural testing - Is our language confusing? Hort Science 21, 215-217

Klougart A (1976) Urea as a nitrogen source for pot plants. Acta Hortic 64, 103-109

Kratky BA, Mishima HY (1981) Lettuce seedling and yield response to preplant and foliar fertilization during transplant production. J Am Soc Hortic Sci 106, 3-7

Magalhaes JR, Wilcox GE (1984) Growth, free amino acids, and mineral composition of tomato plants in relation to nitrogen form and growing media. J Am Soc Hortic Sci 109, 406-411

Marschner H (1986) Mineral nutrition of higher plants. Academic Press, Londres, $674 \mathrm{p}$

Nowak TJ (1986) Foliar fertilizing in pepper with different nutrition in hydroponic cultures. Dev Plant Soil Sci 22, 335-342

Reed DW (1988) Effect of urea, ammonium and nitrate on foliar absorption of ferric citrate. J Plant Nutr 11, $1429-1437$
Swietlik D, Faust M (1984) Foliar nutrition of fruit crops. Hortic Rev 6, 287-355

Tremblay N, Gosselin A (1989a) Growth and nutrient status of celery seedlings in response to nitrogen fertilization and $\mathrm{NO}_{3} / \mathrm{NH}_{4}$ ratio. Hort Science 24, 284-288

Tremblay N, Gosselin A (1989b) Growth, nutrient status, and yield of celery seedlings in response to urea fertilization. Hort Science 24, 288-291

Warncke DD (1986) Analysing greenhouse growth media by the saturation extraction method. Hort Science 21, 223-227

Wilson JB (1988) A review of evidence on the control of shoot: root ratio, in relation to models. Ann Bot $61,433-449$

Zehnalek J, Minar J (1987) Response of sunflower seedlings (Helianthus annuus $\mathrm{L}$ ) to foliar application of solution DAM-390, ammonium nitrate and urea. Scr Fac Sci Nat Univ Purkynianae Brun 17, 203-214 